\section{The current status of the SMH}

The main thrust of Bechara et al.'s arguments is that the $\mathrm{SMH}$ is compatible with our findings and other findings discussed in our paper. We do not entirely disagree. Indeed, we emphasized that 'our findings, together with these other findings in the literature, do not prove that the somatic marker hypothesis is wrong' [2]. Some of the results reviewed above are at odds with apparent predictions of the SMH; however, they are not conclusive. Nevertheless, what we have claimed, and what we think remains clear after an analysis of Bechara et al.'s arguments, is that there is currently no evidence calling for SMs. The key contribution of our findings was to show that SMs are not necessary to explain the results regarding normal participants in the IGT. For all other evidence that Bechara, Damasio, and colleagues have proposed to support the $\mathrm{SMH}$, there are also alternative (and arguably more parsimonious) explanations.

\section{Acknowledgements}

The writing of this article was supported by a fellowship from the Calouste Gulbenkian Foundation (Portugal) to T.V.M. and by NIMH grant MH64445 to J.L.M

\section{References}

1 Bechara, A. et al. (2005) The Iowa Gambling Task and the somatic marker hypothesis: some questions and answers. Trends Cogn. Sci. 9. DOI: 10.1016/j.tics.2005.02.002

2 Maia, T.V. and McClelland, J.L. (2004) A reexamination of the evidence for the somatic marker hypothesis: What participants really know in the Iowa gambling task. Proc. Natl. Acad. Sci. U. S. A. 101, 16075-16080
3 Sutton, R.S. and Barto, A.G. (1998) Reinforcement Learning: An Introduction, MIT Press

4 Bechara, A. et al. (1997) Deciding advantageously before knowing the advantageous strategy. Science 275, 1293-1295

5 Rolls, E.T. (1999) The Brain and Emotion, Oxford University Press

6 Clark, L. et al. (2004) The neuropsychology of ventral prefrontal cortex: Decision-making and reversal learning. Brain Cogn. 55, 41-53

7 Fellows, L.K. and Farah, M.J. (2005) Different underlying impairments in decision-making following ventromedial and dorsolateral frontal lobe damage in humans. Cereb. Cortex 15, 58-63

8 Bechara, A. et al. (2000) Emotion, decision making and the orbitofrontal cortex. Cereb. Cortex 10, 295-307

9 Rolls, E.T. et al. (1994) Emotion-related learning in patients with social and emotional changes associated with frontal lobe damage. J. Neurol. Neurosurg. Psychiatry 57, 1518-1524

10 Fellows, L.K. and Farah, M.J. (2003) Ventromedial frontal cortex mediates affective shifting in humans: evidence from a reversal learning paradigm. Brain 126, 1830-1837

11 Rolls, E.T. et al. (1983) Responses of striatal neurons in the behaving monkey: 1 . Head of the caudate nucleus. Behav. Brain Res. 7, 179-210

12 Ongur, D. and Price, J.L. (2000) The organization of networks within the orbital and medial prefrontal cortex of rats, monkeys and humans. Cereb. Cortex 10, 206-219

13 Killcross, S. et al. (1997) Different types of fear-conditioned behaviour mediated by separate nuclei within amygdala. Nature $388,377-380$

14 Hornak, J. et al. (2004) Reward-related reversal learning after surgical excisions in orbito-frontal or dorsolateral prefrontal cortex in humans. J. Cogn. Neurosci. 16, 463-478

15 Dias, R. et al. (1996) Dissociation in prefrontal cortex of affective and attentional shifts. Nature 380, 69-72

$1364-6613 / \$$ - see front matter (c) 2005 Elsevier Ltd. All rights reserved. doi:10.1016/j.tics.2005.02.006

\title{
Developmental changes in the linguistic brain after puberty
}

\author{
Alice M. Proverbio ${ }^{1}$ and Alberto Zani $^{2}$ \\ ${ }^{1}$ University of Milano-Bicocca, Piazza dell'Ateneo Nuovo 1, 20126 Milan, Italy \\ ${ }^{2}$ Institute of Molecular Bioimaging and Physiology, National Research Council (CNR), Via Fratelli Cervi 93, \\ 20090 Segrate (Milan), Italy
}

The development of reading skills is a complex and very long-lasting process. In an influential study Booth et al. demonstrated age-related changes in the activation of a network of left hemisphere regions, including the inferior frontal area, the superior temporal gyrus, and the angular gyrus. Interestingly, they found that the angular gyrus, which is involved in the mapping between phonological and orthographic representation, is automatically activated in adults during visual orthographic tasks not requiring this operation.

Corresponding authors: Proverbio, A.M. (mado.proverbio@unimib.it), Zani, A. (alberto.zani@ibfm.cnr.it).
Reading relies on the fast and accurate conversion of orthographic characters into their phonological representations. As a result of years of training and exposure to written and spoken language, skilled readers have faster and automatic access to phonology when reading and to orthography when listening [1,2]. Although this might seem obvious, the mechanisms involved in the development of reading skills have still not been thoroughly investigated. Neuroimaging research has provided useful information about which brain regions are active during reading in adults (see Figure 1). These same areas seem to be abnormally activated in dyslexic children [3]. However, the effects of post-pubertal brain maturation and extensive education remain largely 


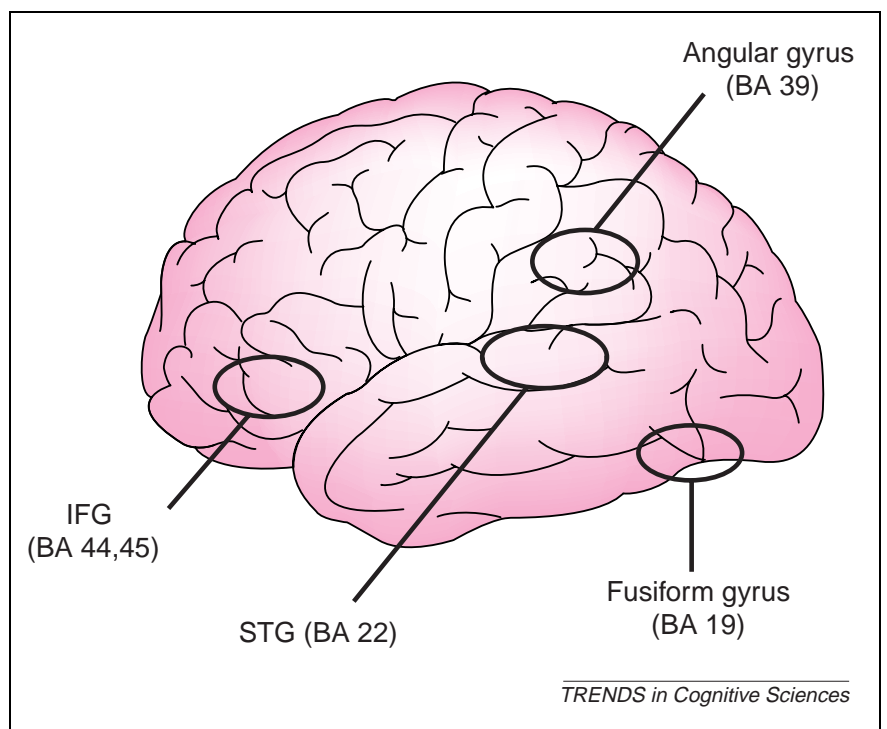

Figure 1. Localization of left hemisphere regions active during reading: the visual word form area (VWFA) in the fusiform gyrus is involved in orthographic processing, the superior temporal gyrus (STG) is involved in phonological processing (input), the inferior frontal gyrus (IFG) is involved in phonological processing (output), and the angular gyrus (AG) is involved in the mapping between phonological and orthographic representation.

unexplored. This gap has been partly filled by Booth and colleagues. They carried out a series of experiments to investigate developmental differences in the neurocognitive networks for linguistic processing in adults, and pubescent children with well-established linguistic competence. They found substantial age-related differences in the activation of several left hemisphere regions, among which the angular gyrus (AG), most interestingly, showed developmental increases in functional activation and automaticity.

The same authors' previous studies had shown that children activate phonological information later and less automatically than adults during reading $[1,2]$. In addition, children show fewer interaction effects between orthographic and phonological representations of words [4] (for example, when making decisions about whether visually similar or dissimilar pairs of letters rhyme). Because they had previously shown that better performance on tasks requiring grapheme-to-phoneme conversion was associated in adults with a greater activation of the supra-marginal/angular gyrus, the authors assumed that children, being less skilled than adults, would show less activation of the same regions.

\section{Neural circuitry involved in rhyme and spelling tasks}

To shed some light on this matter, Booth et al. [5] carried out an fMRI study in which 15 adults and 15 children (9-12-year-olds) performed tasks involving phonological processing (spelling and rhyming judgments) and nonlinguistic control tasks in either the visual or auditory modality. Table 1 shows some examples of words used in the various conditions.

The visual orthographic tasks did not require access to phonological information, and the rhyming auditory ones did not require orthographic information (hence these are 'intramodal' tasks). Conversely, the visual orthographic rhyming tasks required access to
Table 1. Examples of words (with phonetic transcription) used in the various tasks in the study by Booth et al. [5]

\begin{tabular}{|lll|}
\hline $\begin{array}{l}\text { Task: visual or } \\
\text { auditory }\end{array}$ & Similar orthography & Dissimilar orthography \\
\hline Rhyming & SEAT [sit] & HOME [həum] \\
& HEAT [hit] & FOAM [fəum] \\
Spelling & VOTE [vəvt] & SOAP [səup] \\
& NOTE [nəvt] & HOPE [həup] \\
\hline
\end{tabular}

phonological information and the auditory spelling tasks required orthographic information ('crossmodal' tasks). The authors hypothesized that there would be substantial differences between adults and children, in particular in the crossmodal tasks, which require activation of a mechanism providing a strict mapping between phonological and orthographic information. Overall, the adults showed greater activation of the left inferior frontal gyrus (IFG) than did the children in all linguistic tasks. This is consistent with the literature showing age-related increases in the activation of this area during various tasks of noun or verb generation, fluency and reading (e.g. [6]). Neuroimaging studies suggest that the left IFG has a role in processing word phonology in tasks involving rhyme judgments [7] or word vs. pseudo-word reading [8]. The right IFG was also more activated in adults than in children during the visual and auditory spelling tasks (consistent with Booth et al. [9]), and the visual rhyming task. Moreover, both adults and children showed greater activation of the left than the right fusiform gyrus during the visual tasks, thus supporting the view that this area might correspond to the visual word form area (VWFA) [10], and of the left than the right superior temporal gyrus (STG) during the auditory tasks, compatible with the idea that this area is crucial in phonological processing $[11,12]$.

\section{The angular gyrus mediates access to heteromodal linguistic information}

Interestingly, both children and adults showed activation of the left STG during the visual rhyming task that required access to phonological information, and activation of the VWFA during the auditory spelling task that required access to orthographic information. Scalp electrophysiological recordings reveal a selective response of the VWFA to phonemic incongruity at about $185 \mathrm{~ms}$ poststimulus [13]. This finding provides the possible timing for the activation of VWFA in the auditory spelling task. Moreover, Booth et al. [5] showed a manifest greater activation of the left AG in adults than in children in crossmodal tasks (auditory spelling and visual rhyming), indicating that the left AG is involved in these processes (see Figure 2).

Before concluding this section it must be noted that although some authors have observed increased activation of the AG during combined processing of visual and phonological word-form information [5,9,14], other groups have failed to provide supporting evidence for this hypothesis (e.g. $[8,15])$. 
(a)

\begin{tabular}{|c|c|c|c|c|}
\hline Voxels & 89 & 35 & 34 & 27 \\
$x, y, z$ & $-30-6945$ & $-30-6336$ & $-51-5712$ & $-42-4548$ \\
\hline
\end{tabular}

(b)

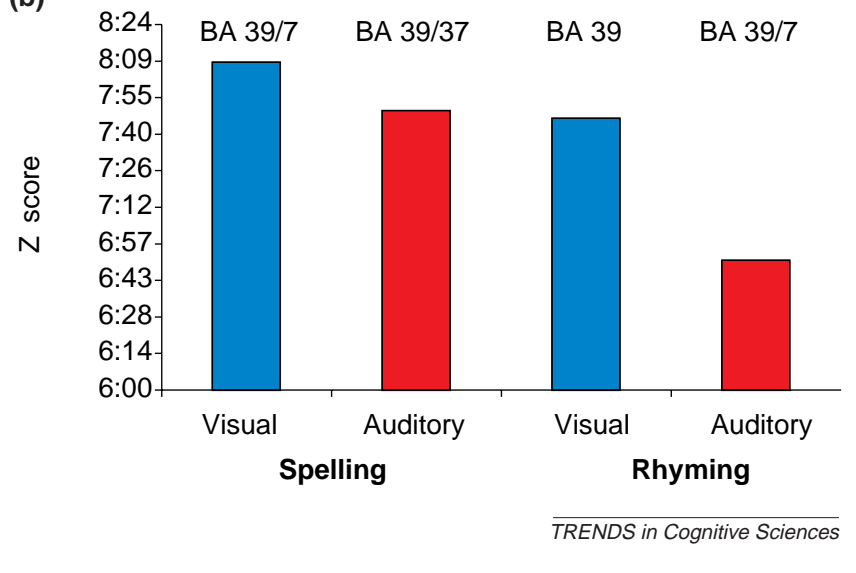

Figure 2. Significant differences in brain activation between adults and children during the auditory and visual tasks used by Booth et al. [5], shown as (a) voxels; (b) $Z$ scores; averaged adult minus child scores in the activation of the AG and neighboring areas (i.e. superior parietal lobule and middle temporal gyrus). The tasks involved the sequential presentation of three stimuli and subjects were required to determine whether the third stimulus matched one of the previous ones on the basis of either spelling (visual) or rhyme (auditory).

\section{Cerebral maturation leads to the automatization of reading routines}

Undoubtedly the most important result found by Booth et al. [5] is that in adults the left AG was more activated in crossmodal than in intramodal tasks, thus suggesting that this area is involved in phoneme-grapheme mapping. Furthermore, in intramodal tasks not requiring access to heteromodal information (e.g. in the visual spelling task, see Figure 2, first column) the AG was significantly more activated in adults than in children. Altogether, these data indicate that skilled readers have automatic access to phonological information during reading $[1,2]$.

The evidence provided by Booth et al. [5] that the phoneme-grapheme mapping system is activated in adults independently of sensory modality and task requirements also indicates a prevalence of automatic versus controlled processes in adults compared with young readers. This may correlate with maturational differences in synaptogenesis, glucose metabolism, myelination and cortical white matter increases occurring between puberty and adulthood (10-20 years), especially in prefrontal areas. Booth et al. [16] have suggested a strict correlation between the development and specialization of neural systems devoted to reading after puberty, which would lead to an improvement in the transmission of neural signals and in the interconnection of brain regions involved in reading. According to this hypothesis, brain maturation, along with practice and exposure to appropriate inputs, would lead to specialization of a network of left brain regions involved in reading. Within this network, the angular or supramarginal gyrus might play a crucial role in the interconnection between heteromodal regions in the mature reader [5].

\section{Does the development of the visual word form area end at puberty?}

The findings of Booth et al. [5] demonstrate developmental changes in brain activation after puberty. One of the questions still left open is whether these changes homogeneously concern all neural structures or whether there are age-related functional differences between areas.

In this regard, Turkeltaub et al. [17] carried out an fMRI study on subjects aged between 6 and 22 years. They found a linearly progressive increase in the activation of the left IFG and middle temporal cortices, as well as a disengagement of the right inferior temporal cortex as age and reading ability increased from early childhood to adulthood. However, they did not observe significant changes in the activity of VWFA across ages. By contrast, other studies have suggested a dramatic development and specialization of this region in the first years of learning to read (5-9 years). Moreover, studies on dyslexic children $[18,19]$ demonstrated that reading skills are positively correlated with the magnitude of activation in the left occipito-temporal region. Evidence for the development of the VWFA after puberty is unclear. For example, Booth et al. [16] reported a greater activation of left BA 19 and 37 in adults than in young adolescents for processing written word forms, but were unable to replicate these findings in the later studies. Therefore, it is still unclear at this point whether the maturation of VWFA ends with puberty or undergoes subsequent functional modifications due to cerebral maturation or expertise.

\section{Summary and future perspectives}

An important issue in developmental cognitive neuroscience is whether the activation of brain systems specialized in reading changes throughout or after the period of acquisition of this skill. Booth et al. [5] have demonstrated that these changes continue even after puberty, accompanying the refinements of reading ability throughout adolescence. In neural terms these changes are associated with a greater activation of a network of left hemisphere regions, including the IFG and STG. Further research is needed to clarify the role of age- and skill-related changes in VWFA functionality. For example, Shaywitz et al. [18] found that reading skill was associated with more activation in the left fusiform gyrus, but they did not find an association with age. Indeed, the real challenge is to disentangle skill from age effects. One way this question could be addressed is to measure brain activation of readers with a different degree of education (for equivalent cerebral maturation), or of different ages but a similar degree of education.

Another aspect that needs further investigation is the precise role of the AG in linguistic processing. Indeed, besides phonemic-orthographic mapping, AG seems deeply involved in semantic processing [15]. The general question is whether the age-related increase in activation of $\mathrm{AG}$ (as well as of IFG) might be related to significant changes in the conceptual system and lexical knowledge of adults compared with children. To tap into different representational systems across ages one might use multiple tasks with parametric manipulations (such as the word/pseudoword contrast). 


\section{Acknowledgements}

We wish to thank the Editor, Shbana Rahman, and the anonymous referees, for their very helpful and perceptive suggestions. We are also very grateful to Patricia Klaas for her valuable comments on the manuscript.

\section{References}

1 Booth, J.R. et al. (1999) Quick, automatic, and general activation of orthographic and phonological representations in young readers. Dev. Psychol. 35, 3-19

2 Booth, J.R. et al. (2000) The association of rapid temporal perception with orthographic and phonologic processing in reading impaired children and adults. Sci. Stud. Reading 4, 101-132

3 Shaywitz, B.A. et al. (1998) Functional disruption in the organization of the brain for reading in dyslexia. Proc. Natl. Acad. Sci. U. S. A. 95, 2636-2641

4 Zecker, S.G. (1991) The orthographic code: developmental trends in reading-disabled and normally-achieving children. Ann. Dyslexia 41, 179-192

5 Booth, J.R. et al. (2004) Development of brain mechanisms for processing orthographic and phonologic representations. J. Cogn. Neurosci. 16, 1234-1249

6 Schlaggar, B.L. et al. (2002) Functional neuroanatomical differences between adults and school-age children in the processing of single words. Science 296, 1476-1479

7 Shaywitz, B.A. et al. (1995) Sex differences in the functional organization of the brain for language. Nature 373, 607-609

8 Hagoort, P. et al. (1999) The neural circuitry involved in the reading of German words and pseudowords: a PET study. J. Cogn. Neurosci. $11,383-398$
9 Booth, J.R. et al. (2003) Relation between brain activation and lexical performance. Hum. Brain Mapp. 19, 155-169

10 Cohen, L. and Dehaene, S. (2004) Specialization within the ventral stream: the case for the visual word form area. Neuroimage 22, 466-476

11 Rumsey, J.M. et al. (1997) Phonological and orthographic components of word recognition. A PET-rCBF study. Brain 120, 739-759

12 Poldrack, R.A. et al. (2001) Relations between the neural bases of dynamic auditory processing and phonological processing: evidence from fMRI. J. Cogn. Neurosci. 13, 687-697

13 Proverbio, A.M. and Zani, A. (2003) Time course of brain activation during graphemic/phonologic processing in reading: an ERP study. Brain Lang. 87, 412-420

14 Howard, D. (1992) The cortical localization of the lexicons: positron emission tomography evidence. Brain 115, 1769-1782

15 Binder, J.R. et al. (2003) Neural correlates of lexical access during visual word recognition. J. Cogn. Neurosci. 15, 372-393

16 Booth, J.R. et al. (2001) The development of specialized brain systems in reading and oral language. Neuropsychol. Dev. Cogn. C Child Neuropsychol. 7, 119-141

17 Turkeltaub, P.E. et al. (2003) Development of neural mechanisms for reading. Nat. Neurosci. 6, 767-773

18 Shaywitz, B.A. et al. (2002) Disruption of posterior brain systems for reading in children with developmental dyslexia. Biol. Psychiatry 52, $101-110$

19 Shaywitz, B.A. et al. (2004) Development of left occipitotemporal systems for skilled reading in children after a phonologically-based intervention. Biol. Psychiatry 55, 926-933

1364-6613/\$ - see front matter (c) 2005 Elsevier Ltd. All rights reserved. doi:10.1016/j.tics.2005.02.001

\title{
Right, left or both? Brain hemispheres and apraxia of naturalistic actions
}

\author{
Raffaella Ida Rumiati
}

Settore di Neuroscienze Cognitive, Scuola Internazionale Superiore di Studi Avanzati, Trieste, Italy

\begin{abstract}
Neuropsychology allows us to see more clearly how brain processes are organized by looking at the way they fall apart. For example, a hundred years of research on brain-damaged individuals with deficits of higher-level motor abilities have shed light on the organization of normal motor abilities. A consistent finding has been the dominant role of the left hemisphere in motor control in right-handers. However, the recent paper of Hartmann et al. challenges this tradition by suggesting that both hemispheres can contribute to complex object and tool use.
\end{abstract}

In everyday life humans skilfully use a very large range of objects and tools. It has often been reported, though, that after left-brain damage the ability to use them can be dramatically reduced in right-handed individuals. The recent paper by Hartmann, Goldenberg, Daumüller and Hermsdörfer [1] seems to suggest that the right hemisphere can also contribute to successful tool use.

Corresponding author: Rumiati, R.I. (rumiati@sissa.it).
Ideational apraxia as a left-brain-damage syndrome

Over the years, the specific deficit of object and tool use has been given different labels such as ideational apraxia [2,3], agnosia [4] or amnesia of object use [5], and conceptual apraxia [6], each corresponding to different accounts. However, all these readings of the deficit share the view that it is conceptual in nature. Recently, some patients have been described with dramatic difficulties in using objects in everyday activities but spared lexical semantic knowledge about them $[7,8]$. By contrast, patients with semantic impairment but spared object use have also been reported [9]. Taken together these observations suggest a relative independence of the action and semantic domains [10].

To distinguish it from a semantic deficit proper, it has been proposed that ideational apraxia might be due to a malfunctioning of the contention scheduling system (see Box 1), the object representation either triggering the incorrect schemas or being disconnected from action schemas when a goal-directed action is attempted $[8,11]$. In a functional imaging study in healthy volunteers, the 